\title{
Identification of Te alloys with suitable phase change characteristics
}

\author{
Ralf Detemple, ${ }^{\text {a) }}$ Daniel Wamwangi, and Matthias Wuttig \\ I. Physikalisches Institut der RWTH Aachen, 52056 Aachen, Germany \\ Gustav Bihlmayer \\ Institut für Festkörperforschung, Forschungszentrum Jülich, 52425 Jülich, Germany
}

(Received 17 March 2003; accepted 7 July 2003)

\begin{abstract}
At present, the discovery and development of phase change materials is mainly based upon empirical strategies and trial and error approaches. Here, we present a structural criterion that needs to be met to enable the mandatory fast recrystallization with sufficient optical contrast that characterizes suitable phase change materials. Comparing the behavior of $\mathrm{AgInTe}_{2}$ and $\mathrm{AgSbTe}_{2}$ films it is demonstrated that only the $\mathrm{AgSbTe}_{2}$ films, which show a cubic coordination, have sufficient density contrast, and hence, also optical contrast to allow phase change recording. (c) 2003 American Institute of Physics. [DOI: 10.1063/1.1608482]
\end{abstract}

Rewritable optical data storage based upon reversible phase transformations utilizes a focused laser to switch between an amorphous and a crystalline state. ${ }^{1,2}$ The information is written by locally melting the crystalline material followed by a rapid cooling, which results in an amorphous bit. Erasing is achieved by heating this amorphous region above the crystallization temperature. Two key attributes are crucial to meet the demands on phase change materials. A sufficient optical contrast between the amorphous and crystalline state is necessary to read the stored information. Furthermore, a fast recrystallization of the amorphous region is mandatory since this is the slowest process involved, which determines the maximum data transfer rate. Unfortunately, no clear microscopic criteria for suitable materials, such as a requirement in terms of composition or crystalline structure have been developed as yet. Hence, the improvement of phase change media is still mainly based on trial and error strategies and empirical rules. ${ }^{3}$ In this letter, on the contrary, a mandatory requirement for a successful storage material based upon its structure will be presented. Understanding and employing this criterion should facilitate a more rapid materials development.

Previously, it had been shown that alloys containing Ag, $\mathrm{In}, \mathrm{Sb}$, and Te are particularly suitable for fast rewritable optical data storage. ${ }^{4}$ Alloys of these elements frequently decompose upon prolonged heating into $\mathrm{AgInTe}_{2}$ and $\mathrm{AgSbTe}_{2} \cdot{ }^{5}$ Hence, we have prepared alloy films of these two materials and investigated their structural, optical and electrical properties; analyzed their recording behavior and performed band-structure calculations of the ground states using density functional theory (DFT) to understand the measured properties.

Both ternary compositions were prepared using thermal evaporation. Si wafers and glass slides, kept at ambient temperature, were selected as substrates, leading to ternary alloys with an amorphous structure. A chemical analysis yielded a composition of $\operatorname{Ag}(23), \operatorname{In}(27), \mathrm{Te}(50)$ where the numbers in brackets denote atomic \%, thereafter called

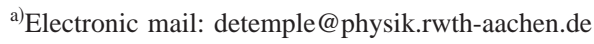

$\mathrm{AgInTe}_{2}$. The properties of $\mathrm{AgSbTe}_{2}$ were examined using two samples, with a stoichiometry of $\mathrm{Ag}(16), \mathrm{Sb}(29), \mathrm{Te}(55)$, and $\mathrm{Ag}(32), \mathrm{Sb}(22)$, and $\mathrm{Te}(46)$.

In a first series of experiments the laser induced initial crystallization of the as-deposited amorphous films was analyzed. Subsequently, also, the amorphization of crystalline films and the recrystallization of amorphous bits were analyzed. In these experiments a laser beam with a focus size of $0.46 \mu \mathrm{m}$ at the sample position was utilized where the laser power was varied between 0 and $30 \mathrm{~mW}$, and the pulse length ranged between $10 \mathrm{~ns}$ and $1 \mathrm{~s}$. On films of both compositions, which had a thickness of $57 \mathrm{~nm}\left(\mathrm{AgInTe}_{2}\right)$ respective $56 \mathrm{~nm}\left(\mathrm{AgSbTe}_{2}\right)$, ablation was observed for long pulses and/or high powers. Ablative hole formation leading to a decrease of film reflectance was confirmed by atomic force microscopy. ${ }^{6}$ However, an increase of film reflectance, which is characteristic for crystallization was only observed for the $\mathrm{AgSbTe}_{2}$ films, while no such reflectance change was observed for the $\mathrm{AgInTe}_{2}$ films no matter how long and/or intense the laser beam was. This either implies that crystallization was not possible or that the change in reflectance for this material is smaller than the smallest measurable reflectance change of our tester, which is smaller than 0.01 . To clarify this situation, measurements of the electrical resistivity of the films were performed while heating the films in an Ar ambient. These data are displayed in Fig. 1. Both films show a pronounced decrease of resistivity upon heating, which is characteristic for crystallization as confirmed by our $\mathrm{x}$-ray diffraction (XRD) analysis. The $\mathrm{AgInTe}_{2}$ sample shows a sharp crystallization at $149^{\circ} \mathrm{C}$, whereas $\mathrm{AgSbTe}_{2}$ exhibits a continuous phase transition starting at $75^{\circ} \mathrm{C}$. It can be, therefore, concluded that laser induced crystallization should be possible also in $\mathrm{AgInTe}_{2}$ films, which indicates that this alloy might only show weak optical contrast between the amorphous and crystalline states.

The higher overall resistivity of the $\mathrm{AgInTe}_{2}$ sample is, furthermore, evidence for a different band gap, which also points towards different optical properties for both materials. This was confirmed by optical spectroscopy and spectroscopic ellipsometry. The measured data were reproduced by modeling the dielectric functions of both $\mathrm{AgInTe}_{2}$ and 


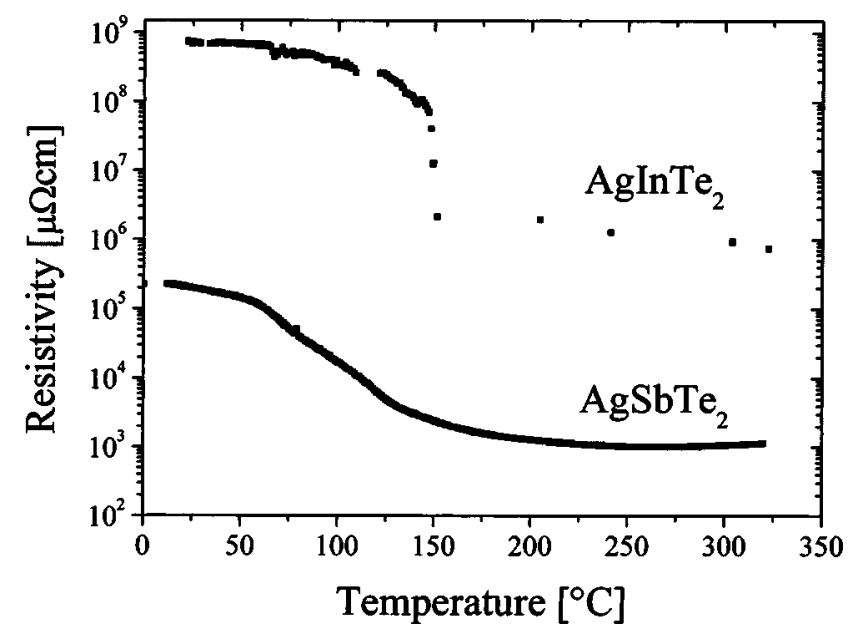

FIG. 1. Electrical resistivity as a function of temperature. The upper curve shows a sudden drop of the electrical resistivity at $149^{\circ} \mathrm{C}$ for $\mathrm{AgInTe}_{2}$, whereas the $\mathrm{Sb}$-containing alloy shows a more continuous phase transition. The transition towards the crystalline phase starts at $75^{\circ} \mathrm{C}$, which was confirmed by x-ray diffraction (not shown here).

$\mathrm{AgSbTe}_{2}$. The two most important results of this analysis were a much higher absorption of the $\mathrm{AgSbTe}_{2}$ films as compared with the $\mathrm{AgInTe}_{2}$ films and a larger change of optical properties between the amorphous and crystalline states for $\mathrm{AgSbTe}_{2}$ than for $\mathrm{AgInTe}_{2}$. This is displayed in Fig. 2 where the calculated change of normalized reflectance for a $60 \mathrm{~nm}$ thin film on glass as a function of the wavelength is plotted for $\mathrm{AgInTe}_{2}$ and $\mathrm{AgSbTe}_{2}$ based upon the dielectric functions determined for the two states of the two alloys. The experimentally observed small reflectance change for $\mathrm{AgInTe}_{2}$ is in good agreement with the corresponding value calculated from the dielectric functions for both phases. It can, hence, be concluded that it is the missing optical contrast between the amorphous and crystalline states of the $\mathrm{AgInTe}_{2}$ sample which renders it useless for rewritable optical data storage. To understand this phenomenon the density and thickness change upon crystallization were determined. The corresponding data are displayed in Fig. 3, where all values have been normalized with respect to the density and thickness of the as-deposited film. The crystallization is ac-

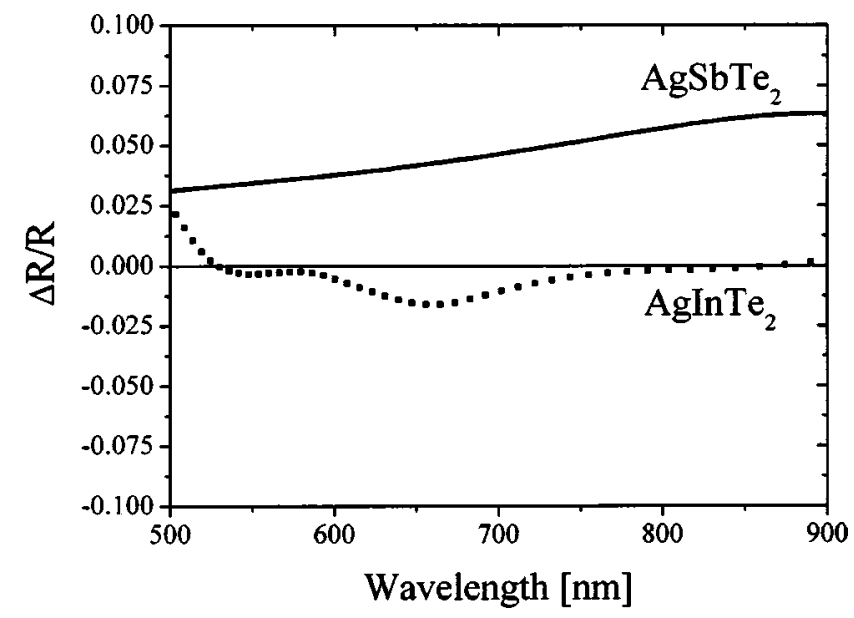

FIG. 2. Normalized reflectance change for both alloys determined from 500 up to $900 \mathrm{~nm}$. The dotted line denotes the contrast for a $60 \mathrm{~nm}$ thin $\mathrm{AgInTe}_{2}$ film on a glass substrate as a function of the wavelength, while the same layer stack, where $\mathrm{AgInTe}_{2}$ is replaced by $\mathrm{AgSbTe}_{2}$, yields the solid line. Downloaded 21 Dec 2006 to 134.94.122.39. Redistribution subject

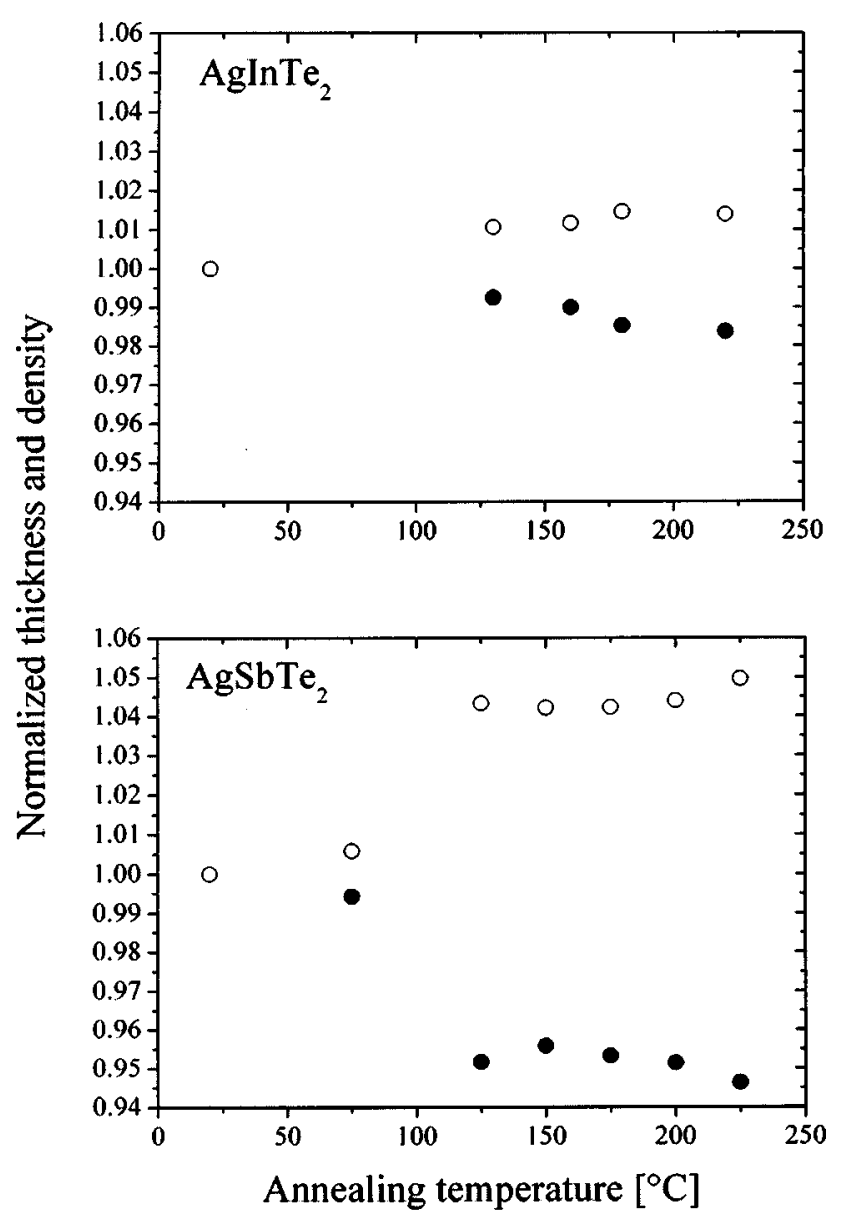

FIG. 3. Thickness change upon crystallization for both ternary alloys as a function of annealing temperature. The thickness is normalized with respect to the as deposited amorphous film, which is $56 \mathrm{~nm}$ for $\mathrm{AgInTe}_{2}$ and $57 \mathrm{~nm}$ for $\mathrm{AgSbTe}_{2}$. The upper diagram shows the small density change of $\mathrm{AgInTe}_{2}$, which is only $29 \%$ compared to the $\mathrm{AgSbTe}_{2}$ alloy.

companied by a reduction of film thickness and a corresponding increase of density by $1.4 \%$ for $\mathrm{AgInTe}_{2}$ and by $4.8 \%$ for $\mathrm{AgSbTe}_{2}$.

This implies that there is a clear correlation between the size of the density change and the magnitude of the change in optical properties between the amorphous and crystalline state. This statement is corroborated by experimental results obtained from GeSbTe and AgInSbTe alloys: The density change upon crystallization scales monotonously also in this group of materials with the contrast between both phases, which starts with the lowest density change of 5.2\% (contrast: 0.31) for $\mathrm{Ag}_{5.5} \mathrm{In}_{6.5} \mathrm{Sb}_{59} \mathrm{Te}_{29}$ and ends with $9.3 \%$ (contrast: 0.55$)$ for $\mathrm{Ge}_{4} \mathrm{Sb}_{1} \mathrm{Te}_{5}$. The intermediate case of a density change of $6.8 \%$ was observed for $\mathrm{Ge}_{2} \mathrm{Sb}_{2} \mathrm{Te}_{5}$, which results in a contrast of $0.37 .^{7-9}$ All values for the optical contrast were calculated for the technologically important wavelength of $630 \mathrm{~nm}$, which is used in current DVD technologies.

To understand the different behavior of $\mathrm{AgInTe}_{2}$ and $\mathrm{AgSbTe}_{2}$, we have determined the structure of the films by $\mathrm{x}$-ray diffraction and have performed DFT calculations employing the generalized gradient approximation (GGA), ${ }^{10}$ using the FLEUR code based on the full-potential linearized augmented plane-wave method. ${ }^{11}$ XRD reveals that $\mathrm{AgInTe}_{2}$ crystallizes in the chalcopyrite structure while $\mathrm{AgSbTe}_{2}$ has
to AlP license or copyright, see http://apl.aip.org/apl/copyright.jsp 


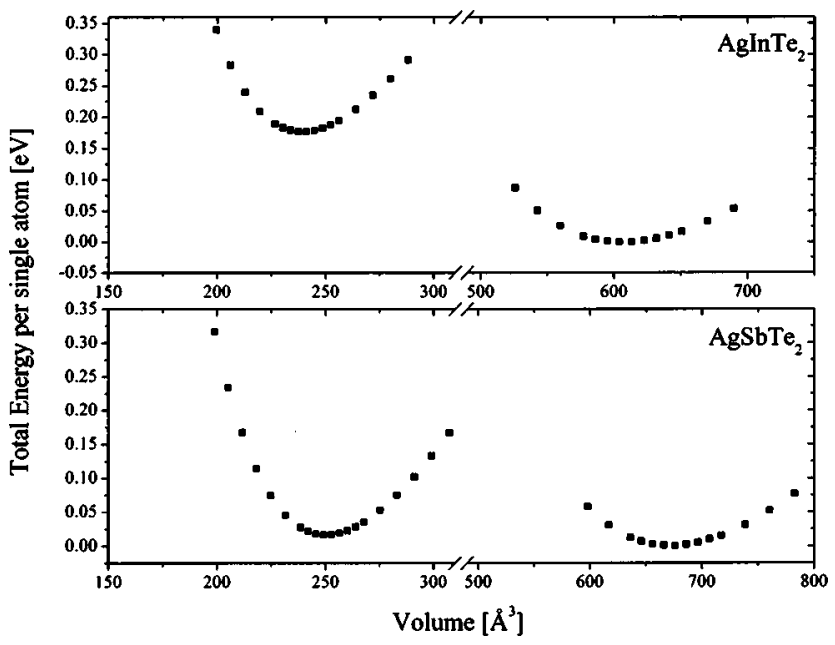

FIG. 4. Variation of total energy per single atom for different configurations. On the left-hand side of both graphs the rocksalt structure is analyzed. The variation of the unit cell of the chalcopyrite structure is displayed on the right-hand side. The upper-view graph shows the dependence for the $\mathrm{AgInTe}_{2}$ phase, whereas $\mathrm{AgSbTe}_{2}$ can be found on the bottom. For the $\mathrm{AgInTe}_{2}$ case, the energy difference between both structures is larger than for $\mathrm{AgSbTe}_{2}$.

the rocksalt structure. Lattice constants of $a=6.435 \AA$ and $c=12.631 \AA$ were determined for $\mathrm{AgInTe}_{2}$, in good agreement with previously reported values $(a=6.419 \AA, c=12.58$ $\AA){ }^{12,13}$ For $\mathrm{AgSbTe}_{2}$ a lattice constant of $6.06 \AA$ was measured, again, in close agreement with the literature value of $6.08 \AA .{ }^{14}$ All lattice constants are reasonably well reproduced by our density functional calculations, as can be seen from Table I. Typically, GGA calculations of heavier elements overestimate the lattice constants by a few percent, as can also be seen in Table I. On the other hand, we can also use DFT to calculate the crystal structure of a hypothetical chalcopyrite structure for $\mathrm{AgSbTe}_{2}$ and a hypothetical lowtemperature rocksalt structure of $\mathrm{AgInTe}_{2}$. These calculations show that the lattice constants for the chalcopyrite structures of $\mathrm{AgInTe}_{2}$ and $\mathrm{AgSbTe}$ vary by less than $4.5 \%$ (see Table I). An even smaller change of the lattice constant is calculated for the rocksalt phase of $\mathrm{AgSbTe}_{2}$ and $\mathrm{AgInTe}_{2}$. Replacing In by Sb in the different phases, hence, only has a small effect on the lattice constants. This can be understood since In and Sb have a very similar covalent radius (1.44 $\AA$ for In and $1.40 \AA$ for $\mathrm{Sb}$ ). The change of ground state structure from chalcopyrite to rocksalt upon replacing In by $\mathrm{Sb}$, hence, is not related to any difference in atomic radii but must rather have an electronic origin. Therefore, we have compared the different energies for the two compositions $\mathrm{AgInTe}_{2}$ and $\mathrm{AgSbTe}_{2}$ in both rocksalt and chalcopyrite structures.

TABLE I. Comparison of the theoretical lattice constants for both structures of $\mathrm{AgInTe}_{2}$ and $\mathrm{AgSbTe}_{2}$. Experimental values are taken from Refs. 12-14.

\begin{tabular}{ccc}
\hline \hline Lattice constant $(\AA)$ & Rocksalt structure (a) & Chalcopyrite structure $(a / c)$ \\
\hline $\mathrm{AgInTe}_{2}$ & $6.22(6.02)$ & $6.84 / 13.12(6.419 / 12.58)$ \\
$\mathrm{AgSbTe}_{2}$ & $6.29(6.08)$ & $7.14 / 13.49(\mathrm{na})$ \\
\hline
\end{tabular}

TABLE II. Comparison of total energies for both structures of $\operatorname{AgInTe}_{2}$ and $\mathrm{AgSbTe}_{2}$.

\begin{tabular}{ccc}
\hline \hline Energy per atom $(\mathrm{eV})$ & Rocksalt structure & Chalcopyrite structure \\
\hline $\mathrm{AgInTe}_{2}$ & 0.177 & 0 \\
$\mathrm{AgSbTe}_{2}$ & 0.0169 & 0 \\
\hline \hline
\end{tabular}

For the different crystallographic arrangements, DFT calculations were performed. The total energy per atom for all possible combinations is shown in Fig. 4 as a function of the unit cell volume. The energy axis is shifted for all curves so that the minimum corresponds to $0 \mathrm{eV}$ for the chalcopyrite structure. In the case of $\mathrm{AgInTe}_{2}$ the chalcopyrite structure is clearly favored, while the situation is more ambiguous for $\mathrm{AgSbTe}_{2}$. The energy differences between both structures are compiled in Table II. Taking into account of the configurational entropy, the rocksalt is preferred to the chalcopyrite structure of $\mathrm{AgSbTe}_{2}$. Hence, DFT calculations do not only reproduce the measured lattice constants but are also able to reproduce the measured ground state structure.

In summary we have shown that sufficient optical contrast between amorphous and crystalline states required for phase change recording can only be found for materials where a sufficient density change accompanies crystallization. This required size of density change is only found for a subset of Te alloys, which crystallize in a phase with cubic or near-cubic coordination. These findings show that the investigated materials exhibit a correlation between the density change upon crystallization and the achieved optical contrast between the amorphous and crystalline phase, where the most pronounced density change in the $\mathrm{Ge}$ - and the Ag-based alloys was observed for materials with a cubic coordination $\left(\mathrm{Ge}_{4} \mathrm{Sb}_{1} \mathrm{Te}_{5}\right.$ and $\left.\mathrm{AgSbTe}_{2}\right)$. This microscopic criterion should help identifying possible candidates for advanced phase change recording. ${ }^{14}$

Financial support by the Fonds der chemischen Industrie is greatly acknowledged. One of the authors (D.W.) acknowledges support by the DAAD.

${ }^{1}$ M. Libera and M. Chen, MRS Bull. 15, 40 (1990).

${ }^{2}$ S. R. Ovshinsky, Phys. Rev. Lett. 21, 20 (1968).

${ }^{3}$ M. H. R. Lankhorst, J. Non-Cryst. Solids 297, 210 (2002).

${ }^{4}$ H. J. Borg, M. v. Schijndel, J. C. N. Rijpers, M. H. R. Lankhorst, G. Zhou, M. J. Dekker, I. P. D. Ubbens, and M. Kuijper, Jpn. J. Appl. Phys., Part 1 40, 1592 (2001).

${ }^{5}$ H. Iwasaki, M. Harigaya, O. Nonoyama, Y. Kageyama, M. Takahashi, K. Yamada, H. Deguchi, and Y. Ide, Jpn. J. Appl. Phys., Part 1 32, 5241 (1993).

${ }^{6}$ V. Weidenhof, I. Friedrich, S. Ziegler, and M. Wuttig, J. Appl. Phys. 86, 5879 (1999).

${ }^{7}$ W. K. Njoroge, H.-W. Wöltgens, and M. Wuttig, J. Vac. Sci. Technol.A 20, 230 (2002).

${ }^{8}$ D. Wamwangi, W. K. Njoroge, and M. Wuttig, Thin Solid Films 408, 310 (2002).

${ }^{9}$ W. K. Njoroge and M. Wuttig, J. Appl. Phys. 90, 3816 (2001).

${ }^{10}$ J. P. Perdew, K. Burke, and M. Ernzerhof, Phys. Rev. Lett. 77, 3865 (1996).

${ }^{11}$ E. Wimmer, H. Krakauer, M. Weinert, and A. J. Freeman, Phys. Rev. B 24, 864 (1981).

${ }^{12}$ H. Hahn, Z. Anorg. Allg. Chem. 271, 153 (1953).

${ }^{13}$ K. J. Range, G. Engert, J. Engels, and A. Weiss, Z. Naturforsch. B 23, 1008 (1968).

${ }^{14}$ J. Res. Natl. Bur. Stand. 25, 47 (1964). 